\title{
Complémentarité des filières sorgho sucré et canne à sucre en Haïti : évaluation des conditions de développement sectoriel d'une innovation
}

\author{
Ludovic Temple ${ }^{1, *}$, Annaïg Levesque ${ }^{2}$, Adnet Lamour ${ }^{3}$, Daphnée Charles ${ }^{4}$ et Serge Braconnier ${ }^{5}$ \\ ${ }^{1}$ UMR innovation, CIRAD, 73 rue JF Breton, 34398 Montpellier, France \\ 2 Inter Aide, 44 rue de la Paroisse, 78000 Versailles, France \\ 3 EMDH/MARNDR, Ferme de Papaye, Hinche, Haïti \\ ${ }^{4}$ Cordaid, 22 Rue Dagena 75 Delmas, Port-au-Prince, Haïti \\ ${ }^{5}$ UMR Agap, CIRAD, Avenue Agropolis, 34398 Montpellier, France
}

\begin{abstract}
Résumé - Nous analysons comment l'introduction du sorgho sucré en Haïti pourrait se traduire en une innovation réussie. L'hypothèse est que le caractère multifonctionnel du sorgho sucré permet de combiner différentes utilisations : alimentaire (graines, fourrages), énergétique (production de bioéthanol ou de gel pour la cuisson et cogénération) et production d'alcool de bouche, dont certaines sont complémentaires de la filière canne. Nous mobilisons un référentiel analytique de filière pour évaluer in itinere les conditions de développement sectoriel d'une introduction variétale. Les données proviennent d'enquêtes à dire d'experts. Les résultats montrent en quoi le fonctionnement de la filière canne à sucre détermine des contraintes spécifiques qui conditionnent le choix des variétés de sorgho. Ils montrent aussi une complémentarité entre la valorisation alimentaire du sorgho sucré sous forme de grain et la production d'alcool. Ils interrogent les conditions de valorisation des grains et des coproduits du sorgho sucré pour l'alimentation animale. Ils invalident enfin son usage énergétique pour la production de bioéthanol.
\end{abstract}

Mots clés : innovation / Haïti / sorgho sucré / filière alimentaire / évaluation d'impact / bioénergie

Abstract - Complementarity of sweet sorghum and sugar cane value chains in Haiti: assessment of the conditions for sectoral development of an innovation. We examine how sweet sorghum implementation in Haiti could be a successful innovation. The hypothesis is that sweet sorghum multiuse would permit the combination of its several uses: in food industry (grain, fodder), energy producing (biofuel or cooking gel and cogeneration) and potable alcohol value chain. We mobilize an analytical frame of reference of value chains to study in itinere the conditions for a sectorial development of an experimental implementation. The data were collected from face-to-face surveys with experts. The results revealed that the functioning of sugar cane industry determines specific constraints, conditioning the choice of sorghum varieties. These results highlight a possible complementarity between the use of sweet sorghum grain for food or feed and its use to produce potable alcohol. They interrogate the potentialities of using sweet sorghum grains and co-products in animal diet. They invalidate the use of sweet sorghum juice for biofuel.

Keywords: innovation system / Haiti / sweet sorghum / food supply chain / impact evaluation / biofuel

\section{Introduction}

En Haïti, pays de 10,4 millions d'habitants, $58 \%$ de la population vit en dessous du seuil de pauvreté. L'agriculture occupe plus de $58 \%$ de la population et contribue à plus de

\footnotetext{
*Auteur de correspondance : ludovic.temple@cirad.fr
}

$22 \%$ du PIB. Cependant, le taux de prévalence de l'insécurité alimentaire, plus de $50 \%$, est l'un des plus élevés du monde (Benoit-Cattin, 2016). La politique agricole est principalement orientée par le dogme libéral depuis les années 1980 (Freguin et Devienne, 2006 ; Paul, 2012): les taxes à l'importation sont passées de 45 à $0 \%$ pour les produits vivriers, sauf pour le maïs $(15 \%)$. La production intérieure diminue alors que les importations alimentaires augmentent. L'essentiel des 
exploitations agricoles sont en polyculture-élevage et mobilisent un matériel rudimentaire (Dufumier, 1988). La canne à sucre est la principale culture génératrice d'emplois salariés dans les activités de transformation. Présente sur une partie importante du territoire, elle est transformée en sirop ou en clairin (rhum local) par des unités artisanales et industrielles. Les superficies en canne ( 80000 ha dans les années 1980) ne représentent plus que 20000 ha depuis les années 2000 et les rendements, environ $35 \mathrm{t} / \mathrm{ha}$, sont les plus bas des Caraïbes (Pierre, 2005). Il ne reste plus que trois grosses distilleries et les importations d'alcool sont en augmentation.

Le sorgho (Sorghum bicolor L. Moench) est une céréale originaire d'Afrique. Il est cultivé en Haïti principalement pour ses grains. Certaines variétés (dites de sorgho sucré) peuvent combiner production de grains et accumulation de sucres dans les tiges. Elles représentent, dans certaines conditions, une alternative à la canne pour produire de l'éthanol, car comme la production de canne, celle de sorgho est mécanisable (Vasilakoglou et al., 2011). Les tiges de sorgho sucré peuvent également, après extraction du jus, représenter un fourrage de bonne qualité. Des travaux posent l'hypothèse que l'exploitation de la multifonctionnalité du sorgho (grain, fourrage, fibres, alcool, énergie) constitue une opportunité de développement, notamment dans les zones sèches et marginales (Chantereau et al., 2013 ; Damasceno et al., 2014; Vinutha et al., 2014). Elle permettrait d'activer une économie circulaire de valorisation industrielle (alimentaire ou énergétique) des coproduits. Cela pour mieux répondre aux besoins de sécurisation alimentaire et énergétique au niveau local ou national.

Principalement cultivé dans les zones marginales et sèches en Haïti, le sorgho grain (non sucré) est la troisième céréale cultivée dans le pays après le riz et le maïs. Il est indispensable à la sécurité alimentaire du pays. Comme la canne, il accuse une baisse des superficies cultivées, qui sont passées de 200000 ha dans les années 1980 à 20000 ha en 2012. Les rendements sont stables depuis 1979 et proches de $800 \mathrm{~kg} / \mathrm{ha}$ (FAOSTAT, 2016). Ce contexte a incité la recherche agronomique à introduire en Haïti des variétés de sorgho sucré. L'objectif étant de caractériser les conditions dans lesquelles ces introductions permettent de générer des innovations qui réalisent des complémentarités technologiques entre la canne à sucre et le sorgho. L'innovation est ainsi assimilée au processus qui met en complémentarité les dimensions techniques, organisationnelles et institutionnelles (Touzard et al., 2014) qui valorisent la multifonctionnalité du sorgho. Cette multifonctionnalité pourrait être au centre d'économies de gamme (synergies de complémentarités entre production) dans le système agraire haïtien. Elle permettrait potentiellement, en renforçant l'intégration de l'agriculture dans les activités agro-industrielles ou agro-artisanales de transformation, de restaurer la compétitivité des productions de canne à sucre et de sorgho.

\section{2 Évaluation in itinere des conditions de faisabilité de l'innovation «sorgho sucré"}

\subsection{Méthode d'analyse du processus d'innovation «filière sorgho sucré "}

Les méthodes d'évaluation d'impact in itinere d'innovation renvoient de manière dominante à la mobilisation de calcul de coûts-bénéfices pour estimer les taux de rentabilité interne des investissements réalisés (Maredia et Raitzer, 2010). Appliquées dans et pour les entreprises, ces méthodes sont peu adaptées pour appréhender les externalités territoriales ou sectorielles, c'est-à-dire les conséquences environnementales, sociales ou économiques non évaluées par les mécanismes marchands. Ces externalités concernent, par exemple, les connaissances et apprentissages technologiques, ou les effets de spillover (effets indirects, externalités d'apprentissages) qui accélèrent ou ralentissent les mécanismes d'adoption d'une technologie. Ainsi, l'augmentation du taux d'adoption d'une technologie se traduit par des rendements croissants, c'est-àdire que son efficacité (rapport entre la production et les investissements nécessaires) augmente plus que proportionnellement par rapport au taux d'adoption (Temple et al., 2016). Dans le cas d'une innovation dont les conditions de réussite sont posées par la multifonctionnalité, l'utilisation de ces méthodes impose que soient mieux identifiées les différentes conditions (micro, méso, macro-économiques) qui permettent d'anticiper l'impact futur. Pour tester en quoi la multifonctionnalité du sorgho sucré présente une potentialité de développement pour le secteur agricole et alimentaire haïtien, nous mobilisons une méthode d'analyse de filière qui explore les différentes conditions critiques et les contraintes ou opportunités déterminantes des mécanismes d'adoption. Ce référentiel méthodologique se construit par la mise en interaction des connaissances générées par différentes disciplines (économie, agronomie, technologie) sur une production donnée (Temple et al., 2011). Il examine l'introduction du sorgho sucré en segmentant l'analyse du processus en trois phases technologiques différentes :

- l'activité de production de sorgho (y compris sucré);

- la transformation en alcool des grains ou bien des jus sucrés extraits des tiges;

- l'utilisation et la commercialisation des coproduits dans l'alimentation animale et la production d'alcool à usage alimentaire ou énergétique.

L'analyse est focalisée sur l'étude des différentes filières qui utilisent le sorgho ou ses coproduits. Elle évalue comment la propriété sucrière du sorgho permettrait son usage industriel comme complément de la canne à sucre pour produire de l'alcool à usage alimentaire ou énergétique. Elle précise ainsi les conditions organisationnelles et techniques qui rendraient compatibles les multiples usages du sorgho sucré au sein du secteur agroalimentaire.

L'ancrage institutionnel de cette étude est localisé dans un projet de recherche développement (S3F-Haiti.cirad.fr) associant des centres de recherche et des entreprises du secteur sucrier.

\subsection{Dispositif de collecte de données et d'informations}

Trois dispositifs d'enquête à dire d'acteurs, mis en place entre 2012 et 2015 par les co-auteurs, sont mobilisés.

Les données secondaires (rapports de projets de développement, statistiques, publications scientifiques, cartes) ont été collectées sur un échantillon total de 70 personnes ressources dans les institutions et entreprises qui structurent le système de recherche et d'innovation haïtien : directions départementales de l'agriculture et mairies, organisations non gouvernementales, 
Tableau 1. Utilisation du sorgho sucré en élevage bovin.

Table 1. Use of sweet sorghum in cattle rearing.

\begin{tabular}{llll}
\hline Atouts (forces) & Contraintes (faiblesses) & Opportunités & Menaces \\
\hline Disponible en période sèche & $\begin{array}{l}\text { Diminution de la qualité du } \\
\text { pâturage en saison sèche }\end{array}$ & Valorisable par les ruminants & $\begin{array}{l}\text { Baisse de la fertilité des sols par } \\
\text { manque de renouvellement de } \\
\text { matière organique si les déjections } \\
\text { des animaux ne sont pas récupérées }\end{array}$ \\
$\begin{array}{l}\text { Les bagasses de sorgho sont une } \\
\text { alternative au gaspillage des tiges } \\
\text { La conservation des bagasses }\end{array}$ & $\begin{array}{l}\text { Gaspillage de la ressource } \\
\text { (feuilles, tiges) }\end{array}$ & $\begin{array}{l}\text { Bagasses ensilées, séchées, } \\
\text { utilisées dans les exploitations }\end{array}$ & $\begin{array}{l}\text { Qualité alimentaire des bagasses extraction du jus } \\
\text { aprèn }\end{array}$
\end{tabular}

La conservation des bagasses

améliore la disponibilité fourragère et de la conservation des

fourrages

Les bagasses de sorgho sont très appréciées par les ruminants
Manque d'équipement pour

broyer et ensiler les bagasses

Faible capacité d'investissement

Temps pour récupérer et

épandre les fumures

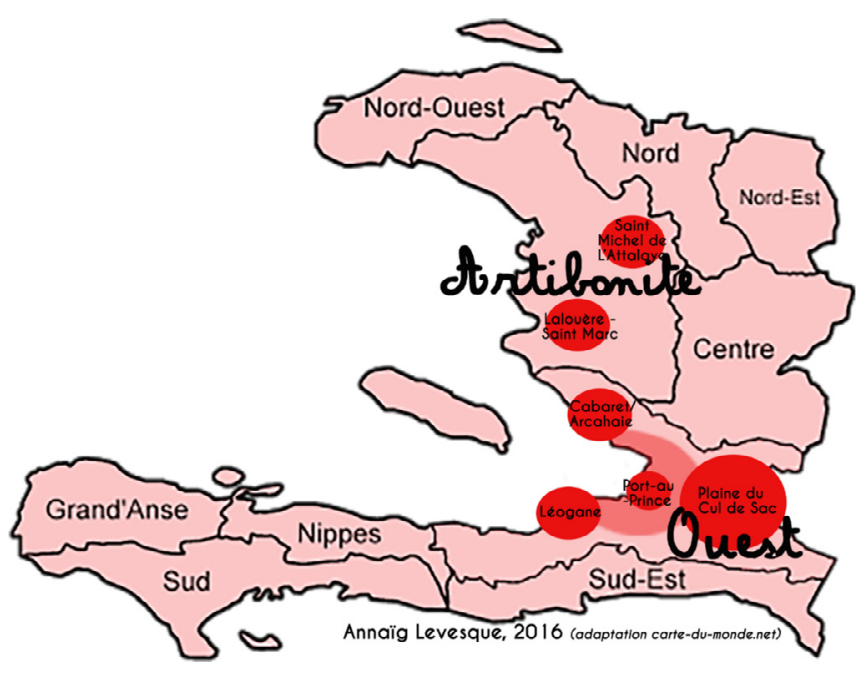

Fig. 1. Localisation géographique des zones de l'étude.

Fig. 1. Map of the study area.

instituts de recherche et d'enseignement, associations d'éleveurs et de producteurs. Les résultats de ces enquêtes ont orienté la méthodologie de collecte des données primaires, réalisée ensuite en face à face auprès d'échantillons d'agriculteurs et d'entrepreneurs des différentes filières considérées.

Des enquêtes par questionnaire en face à face ont permis de collecter des données primaires dans les régions de production de sorgho où sont présentes des entreprises liées au secteur sucrier-distilleries, siroperies, artisanats de rapadou (sucre de canne artisanal brut coagulé dans des moules)-pouvant l'utiliser pour produire de l'alcool. Elles ont été effectuées aussi dans des localités où se trouvent des entreprises produisant des aliments pour animaux (fabricants et/ou importateurs d'aliments). Ces enquêtes ont ciblé trois sites (Fig. 1):

- un site de fabrication de clairin (Saint Marc, Saint Michel de L'Attalaye, Léogane, Cabaret/Arcahaie) avec du sorgho grain non sucré et photopériodique;
- un site de plaine où la culture mécanisée industrielle de la canne à sucre dans de grandes exploitations est dominante (plaine du Cul de Sac), aux portes de Port-au-Prince, où l'on trouve déjà du sorgho sucré avec la variété Papèsèk ; - des unités de production animale (bovine, porcine et avicole), où le sorgho peut constituer une opportunité pour l'alimentation animale.

$\mathrm{Au}$ total, près de 300 producteurs de sorgho et industriels ont été interviewés à l'aide de questionnaires semi-directifs portant sur les systèmes de production, avec un focus prioritaire sur le sorgho et la canne. Ces données ont été synthétisées dans les tableaux 1 et 2 identifiant les atouts et contraintes, opportunités et menaces.

\section{3 État des lieux de la filière sorgho en Haïti : un diagnostic sur données secondaires}

\subsection{Systèmes de production du sorgho}

Le sorgho a été introduit en Haïti au $\mathrm{XVI}^{\mathrm{e}}$ siècle quand l'esclavage a remplacé la main-d'œuvre caraïbe par des populations africaines. Introduit à l'origine dans des zones sèches en contrefort des zones basses ou sur les plateaux agropastoraux, il est aujourd'hui plutôt cultivé dans les plaines sèches et les mornes. Avec une superficie cultivée estimée à 126000 ha, soit 21,3\% des surfaces en céréales (MARNDR, 2012), le sorgho se situe au deuxième rang des cultures derrière le maïs. Sa production annuelle, $105000 \mathrm{t}$, est en diminution. Ainsi, les disponibilités par habitant, de $40 \mathrm{~kg}$ dans les années 1960-1970, ont chuté à $10 \mathrm{~kg}$ depuis les années 1990 (FAOSTAT, 2016). Pourtant, cette céréale reste importante pour la sécurité alimentaire des zones rurales. La récolte a principalement lieu de novembre à janvier. En ville, le sorgho se situe dans les biens qualifiés d'inférieurs et dont la consommation est occasionnelle.

Les agriculteurs interrogés produisent en grande partie du sorgho et dans une moindre mesure de la canne à sucre (respectivement 84 et $53 \%$ pour les 2 premières enquêtes). 
Tableau 2. Utilisation du sorgho sucré dans l'alimentation des porcs et de la volaille.

Table 2. Use of sweet sorghum in feeding pigs and poultry.

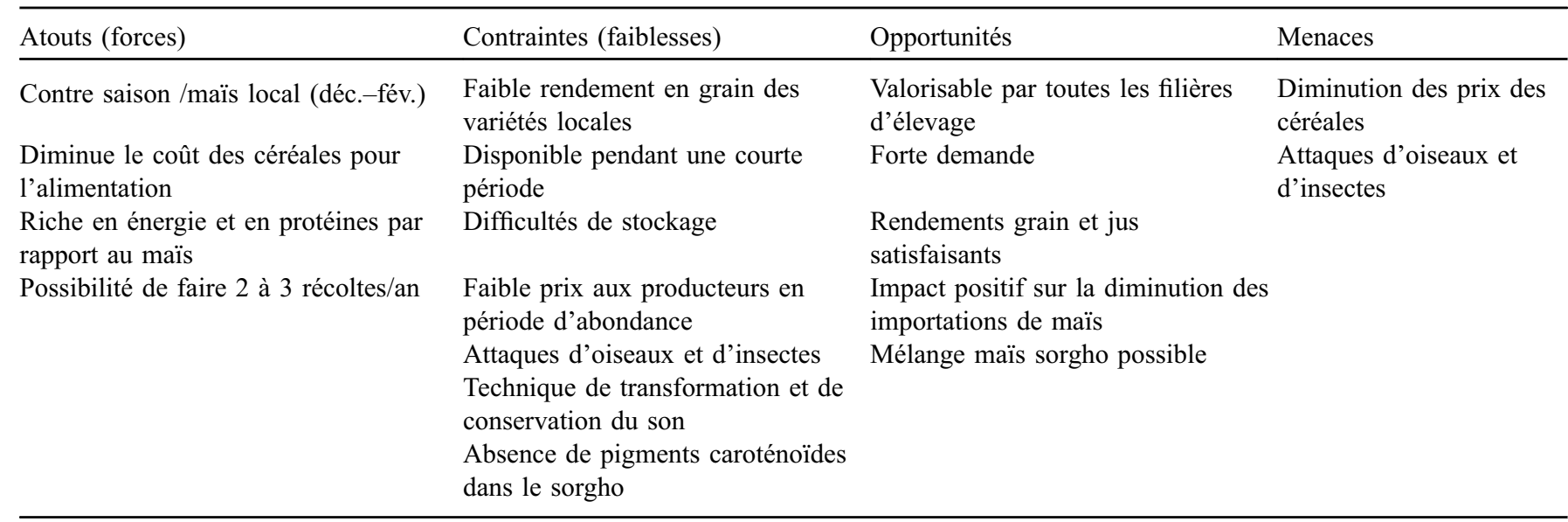

L'élevage des ruminants est pratiqué dans la plupart des exploitations, où les bovins sont utilisés pour le labour et le transport. L'essentiel du sorgho récolté est autoconsommé; une partie est vendue sur des marchés locaux. De faibles parts sont utilisées pour l'alimentation animale et conservées comme semences.

Le sorgho est souvent cultivé en association avec le maïs, le niébé (Vigna unguiculta) et le pois congo (Cajanus cajans), sauf en plaine industrielle où il est en monoculture. Les parcelles sont petites et dispersées.

Depuis 2015, les plantations de sorgho sont attaquées par trois insectes (aphides, pucerons et une mouche blanche), qui sont à l'origine du dessèchement de la plante et de pertes importantes de rendement dans le centre et le sud de l'île.

Les producteurs cultivent en majorité des variétés photopériodiques, dont la floraison est déclenchée par des jours courts (novembre-décembre). Ce caractère synchronise la floraison et donc la récolte de toutes les parcelles et limite ainsi potentiellement les attaques d'insectes et d'oiseaux.

Depuis les années 1990, de nombreux projets ont introduit des variétés de sorgho non photopériodiques, qui ont été adoptées par les agriculteurs dans certaines zones (plaine Arcahaie). L'introduction de variétés de sorgho sucré non photopériodiques, qui permettent de réaliser deux récoltes par an, présente l'inconvénient de concentrer les dégâts des oiseaux sur de faibles superficies lors de la seconde récolte. L'utilisation de ces variétés non photopériodiques impliquerait dans les conditions actuelles soit de massifier les plantations sur de grandes superficies soit de lutter mécaniquement (chasse) ou chimiquement (poisons) contre les oiseaux (enquête à dire d'experts).

\subsection{Transformation et utilisations du sorgho en Haïti}

\subsubsection{Transformation des grains pour la consommation humaine}

La semoule issue du décorticage/broyage (manuel ou mécanique) doit respecter un critère de qualité mentionné par la totalité des personnes interrogées : elle doit être de couleur blanche pour rappeler celle du riz. Le sorgho représente actuellement 10 à $20 \%$ des activités des moulins, contre 25 à $60 \%$ pour le maïs et 30 à $40 \%$ pour le riz.

\subsubsection{Valorisation des coproduits dans l'alimentation animale}

L'agriculture familiale haïtienne repose sur un système d'intégration polyculture-élevage (Pillot, 1992). On compte 500000 exploitations qui ont un à deux bovins par exploitation, et environ 800000 exploitations possédant 2,5 à 3 caprins par exploitation.

\subsubsection{Utilisation des feuilles et repousses par les ruminants}

Après la récolte du sorgho en saison sèche, les feuilles et les tiges laissées sur la parcelle, ainsi que les repousses, servent de vaine pâture aux bovins, caprins et équins. Il en résulte néanmoins une perte de ressource par piétinement. Les tiges sèches non broyées ont une faible appétence et sont peu valorisées. Leur composition nutritive est voisine de celle du maïs au même stade, et leur rendement (tige) est de 10 à $15 \mathrm{t} /$ ha. Elles sont brûlées lors de la préparation du sol et contribuent à sa fertilisation organique.

\subsubsection{Utilisation du son de sorgho pour l'élevage de porcs}

L'élevage porcin est pratiqué par $60 \%$ des exploitations agricoles haïtiennes ( 1 à 3 porcs). Les porcs sont nourris avec des restes de cuisine, des résidus de récolte, du son de maïs, de sorgho ou de blé (acheté). Les grains de sorgho sont utilisés dans la fabrication d'aliments pour porcins quand leur prix est plus bas que celui du maïs. Dans les unités intensives, le son de sorgho est mélangé avec du son de blé et du maïs importés.

\section{Introduction du sorgho sucré en Haïti : conditions de réalisation d'une innovation dans la filière canne}

Entre 1982 et 1988, des variétés de sorgho sucré ont été introduites par l'Organisme de développement du Nord (ODN) pour des objectifs de développement industriel. Elles ont été 
abandonnées du fait de leur faible rendement par rapport à la canne. Dans les années 1990, à St Michel (département de l'Artibonite), la République de Taiwan a introduit de nouvelles variétés auprès de 200 agriculteurs pilotes. Le cycle court de ces variétés (3 à 4 mois au lieu de 6 en moyenne) a été fortement apprécié par les agriculteurs. L'aspect des grains (arrondis, brillants, plus gros) et le port dressé des panicules, en opposition au port retombant des variétés photopériodiques, ont attiré les oiseaux, qui, selon les agriculteurs, «sélectionnaient» la nouvelle variété.

En octobre 2010, l'introduction dans des parcelles expérimentales par le projet (SF3) de plusieurs variétés sucrées provenant de différents programmes de sélection (Centre de coopération en recherche agronomique pour le développement [CIRAD], International Crops Research Institute for the Semi-Arid Tropics [ICRISAT], Empresa Brasileira de Pesquisa Agropecuária [EMBRAPA]) a porté sur 80 variétés. Ces variétés ont été choisies en fonction des connaissances issues de la sélection participative sur les attentes des producteurs, notamment en Amérique centrale (Trouche et al., 2009). À l'issue des tests expérimentaux, quatre variétés ont été retenues pour des études ultérieures. Parmi ces quatre variétés figurait une variété locale sucrée dénommée Papèsèk, déjà présente sur le territoire haïtien. Des travaux complémentaires (Leclerc et al., 2014) ont confirmé les ressemblances entre Papèsèk et Sureño, variété du Honduras qui avait été introduite pour son rendement en grains, mais en ignorant ses qualités sucrières. Il existe donc actuellement des superficies (difficilement quantifiables) déjà cultivées en sorgho sucré, mais non exploitées pour leur potentiel sucrier. Les premiers tests ont montré (Leclerc et al., 2014) que certaines des quatre nouvelles variétés sélectionnées ont un brix (fraction de saccharose contenue dans le jus, exprimée en pourcentage de matière sèche soluble) supérieur à 17. De plus, elles atteignent des rendements en grains supérieurs de 1 à 2 t/ha aux variétés locales.

\subsection{Potentialités d'usage du sorgho sucré dans la filière alcool alimentaire}

Deux types de structures semi-industrielles ou agroartisanales de production d'alcool existent:

- les siroperies, qui sont des unités de transformation de la canne en sirop, qui est stocké avant d'être vendu aux distilleries pour une transformation en alcool. De petites unités (zone de St Michel) produisent aussi du rapadou (sucre non raffiné);

- les guildiveries (distilleries) qui sont des unités de transformation de la canne et/ou du sirop en alcool de bouche (clairin).

La régression de la superficie en canne diminue la rentabilité de ces usines. Selon nos enquêtes, $70 \%$ des unités fonctionnent 10 à $50 \%$ en dessous de leur capacité normale. Le sorgho sucré permettrait d'allonger la période de fonctionnement des usines. En effet, on peut produire de l'alcool (éthanolcarburant) à partir des tiges de sorgho selon les mêmes techniques que celles utilisées pour la canne, tout en ayant aussi la possibilité de produire de l'énergie par combustion des bagasses (Braconnier et al., 2014).
En Haïti, le calendrier cultural du sorgho sucré autorise deux périodes de récoltes par an, l'une avant la récolte de la canne, l'autre après.

L'utilisation du sorgho sucré pour produire de l'alcool permettrait donc d'élargir la période de fonctionnement des usines. Une seconde option serait d'utiliser le sorgho en complément de la canne pour augmenter le taux d'utilisation des usines qui sont actuellement sous-approvisionnées pendant la période cannière du fait de la régression de la canne.

Dans les deux cas, la rentabilité financière des usines s'en trouverait améliorée du fait d'un meilleur amortissement des coûts fixes. La baisse du coût de production de l'alcool qui serait ainsi obtenue pourrait rendre rentable l'usage de l'alcool pour produire de l'énergie (bioéthanol). Ces scénarios impliquent cependant de disposer de variétés adaptées aux conditions climatiques et de volumes de tiges de sorgho suffisants pour une transformation à l'échelle industrielle.

\subsection{Contraintes d'approvisionnement en sorgho des usines de canne}

Les principales contraintes sont liées à la localisation des zones de culture de sorgho sucré et aux conditions de transport et de conservation des tiges. Les parcelles de canne sont cultivées à proximité des unités de transformation, dans les zones irrigables et mécanisables. En revanche, le sorgho se localise sur les terres marginales plus lointaines. La distance du lieu de production du sorgho à l'unité de transformation, ainsi que le mode de transport utilisé, conditionnent les coûts de la matière première rendue à l'usine et donc la rentabilité d'une valorisation par l'industrie cannière.

Une autre contrainte majeure est liée à la courte durée de conservation des tiges de sorgho sucré après la coupe, qui impose une forte proximité entre lieu de production et lieu d'usinage. Cela représente un réel frein pour le sorgho cultivé sur des terres marginales ou par de petits producteurs isolés. Une solution pourrait être de concevoir et d'utiliser des moulins mobiles qui puissent accéder aux parcelles marginales où se cultive le sorgho avec les infrastructures de transport existantes. Tout en diminuant les coûts de transport (moindre volume), cela raccourcirait le délai entre récolte et usinage. L'émergence d'entrepreneurs assurant ce service entre des producteurs peu organisés et les usines fait partie des innovations nécessaires (Fig. 2).

\subsection{Perspectives limitées d'une valorisation dans la filière bioéthanol}

L'éthanol produit à partir du jus sucré de sorgho peut constituer un combustible de substitution au charbon de bois. Ce dernier est en effet la principale source d'énergie pour la cuisson, même si l'utilisation de réchauds à gaz ou à kérosène se développe, respectivement dans 11 et $26 \%$ des ménages à Port-au-Prince. L'usage du bois est responsable de l'accélération de la déforestation et de ses conséquences sur la diminution de la biodiversité, sur l'érosion des sols, voire sur les glissements de terrain. Mais la filière bois est aussi source de revenus dans les zones rurales et sécurise l'accès à l'énergie pour une population pauvre (elle fait vivre 150000 personnes et représente 70 à $80 \%$ de la consommation 


\section{Filière sucrière en plaine du Cul de Sac}

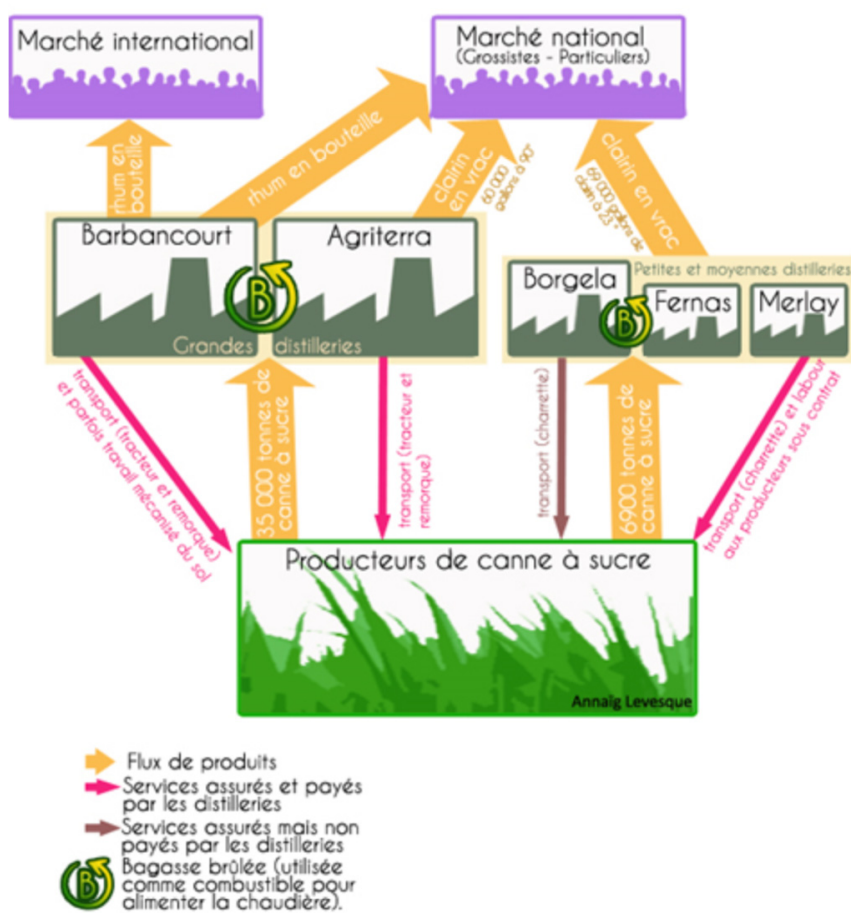

Fig. 2. Structure de la filière sucrière en plaine industrielle du Cul de Sac.

Fig. 2. Structure of sugar cane value chain in Cul de Sac plain.

énergétique du pays). Par ailleurs, des potentialités d'amélioration de son efficacité existent et pourraient atténuer son impact environnemental. L'utilisation d'éthanol comme combustible dans les cuisines exigerait un prix compétitif par rapport aux énergies fossiles (gaz, pétrole) et une adaptation des conditions d'usage dans l'alimentation (réchauds, normes de sécurité, habitudes alimentaires). La production d'un gel se substituant au bois de pin pour l'allumage des foyers à charbon constitue une niche d'innovation qui pourrait être approfondie.

\section{Potentialités d'usage du sorgho sucré dans le secteur de l'élevage}

\subsection{Dans l'alimentation des ruminants}

Les résidus de sorgho local alimentent les ruminants en saison sèche, mais leur valeur nutritive est faible en raison d'une exposition prolongée au soleil et du piétinement lié à la vaine pâture. Les disponibilités sont saisonnières et insuffisantes. Le développement du sorgho sucré pourrait contribuer à accroître et à améliorer la production de fourrage pour l'alimentation animale à partir des bagasses de sorgho.

En effet, l'extraction du jus des tiges de sorgho sucré dans les moulins ou usines génère des bagasses appréciées par les ruminants. Ces bagasses peuvent être consommées rapidement ou stockées pour être disponibles en fin de saison sèche (début de la saison des pluies), lorsque le fourrage est rare. Il y a deux options pour le stockage de la bagasse: sous forme sèche ou d'ensilage. Quelle que soit l'option, elle nécessite des investissements (broyeurs, silos, bâches plastiques) ainsi que la formation des agriculteurs. Ces coûts semblent difficiles à amortir pour de petites exploitations, même avec deux cycles par an. La qualité alimentaire de ces bagasses est de plus incertaine et dépend des techniques d'extraction des jus (Tab. 1).

Par ailleurs, l'enlèvement des tiges hors des parcelles, afin de valoriser les bagasses de sorgho sucré, poserait à long terme le problème du maintien de la fertilité des parcelles. En effet, la diminution de la présence des animaux en vaine pâture et de la restitution au sol des pailles $(75 \%$ des résidus de récolte) réduirait fortement le recyclage de matière organique et d'éléments minéraux (un hectare de résidus de sorgho peut nourrir deux bovins pendant quatre mois de période sèche en valorisant $25 \%$ des résidus de récolte (Dehaynin, 2007). De plus, sur le plan social, la vaine pâture permet à de nombreux petits producteurs de nourrir leurs animaux (une vache ou deux) en saison sèche, sans débours monétaires.

\subsection{Dans l'élevage industriel avicole et porcin}

Les filières d'élevage industriel en Haïti sont les volailles poulets de chair et pondeuses - et les porcs. Pour ces élevages, une contrainte majeure est le coût élevé de l'alimentation, une partie de cette alimentation reposant sur des importations de céréales (maïs). Parallèlement, la valorisation des tiges de sorgho sucré se traduit par une mauvaise qualité des graines de sorgho (stade de maturité, homogénéité...), qui deviennent alors un coproduit potentiel pouvant remplacer en partie le maïs importé pour l'alimentation animale (Fig. 3).

L'utilisation du sorgho dans la formulation de ce type d'aliment reste faible et s'explique par:

- la disponibilité relativement faible en grain de sorgho;

- un différentiel de prix défavorable avec le maïs importé;

- la faible teneur des grains de sorgho en caroténoïdes, qui peut cependant être corrigée par une formulation mixte $(40 \%$ sorgho $+60 \%$ maïs $)$ ou par adjonction de poudre de moringa.

Le tableau 2 présente la matrice SWOT (forces, faiblesses, opportunités, menaces) de l'utilisation des grains de sorgho sucré dans l'alimentation avicole et porcine.

L'introduction du sorgho sucré dans les exploitations agricoles pourrait aussi conduire à l'utilisation du jus et du sirop dans l'alimentation des porcs. Comme le jus de canne, le jus de sorgho sucré est très énergétique et pourrait remplacer l'amidon de céréales. Mais cette substitution potentielle dépend aussi des politiques d'importation du maïs (Tab. 2).

\section{Conclusion}

L'exploration des conditions de développement du multiusage du sorgho sucré en Haïti confirme de réelles potentialités pour améliorer la compétitivité de la filière canne à sucre dans la production d'alcool alimentaire. Son usinage en 


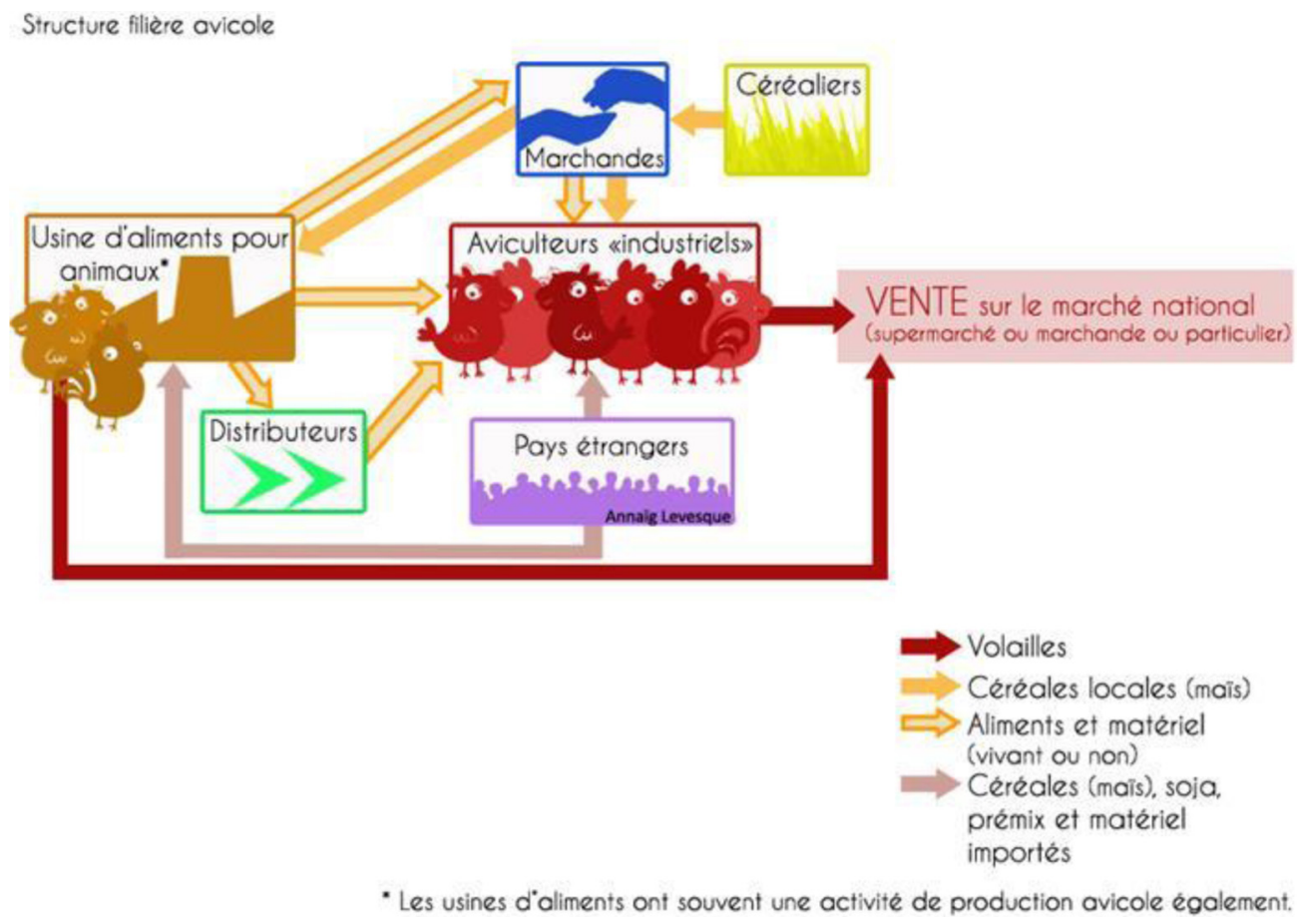

Fig. 3. Structure de la filière avicole.

Fig. 3. Structure of poultry value chain.

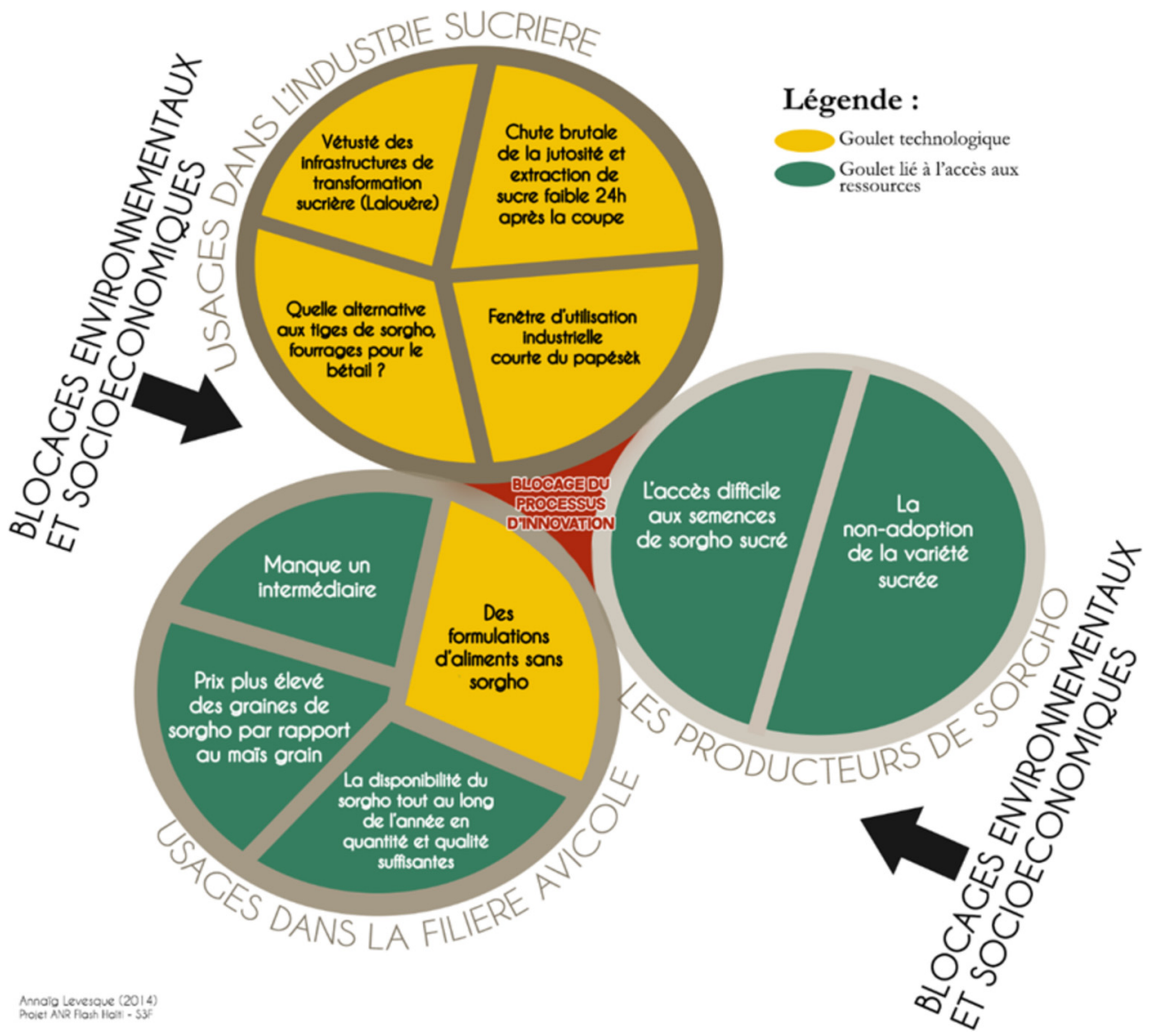

Fig. 4. Facteurs de blocage des filières de valorisation du sorgho sucré.

Fig. 4. Blocking factors of sweet sorghum value chains. 
complémentarité de la canne augmenterait la rentabilité des moulins. La valorisation des coproduits dans l'affouragement en saison sèche ou la valorisation du son dans l'élevage avicole et porcin peut contribuer à renforcer l'intégration agriculture/ élevage. L'usage énergétique de l'éthanol semble quant à lui peu adapté aux conditions haïtiennes (Fig. 4).

D'un point de vue spatial, les zones Arcahaie/Cabaret, plaine du Cul de Sac et St Michel de l'Attalaye offrent les meilleures possibilités d'expansion du sorgho sucré pour un usage industriel. Dans les zones comme Léogane et Saint Marc, avec des zones mal desservies, la valorisation se situe plus dans les usages alimentaires du grain et fourrager des tiges. Une explication de ces différences réside dans les possibilités de mécaniser ou non la récolte du sorgho. Il est donc nécessaire de proposer une diversité de variétés pour cette diversité des contextes.

La réalisation de ces potentialités implique de lever des contraintes technologiques, économiques et sociales que l'évaluation d'impact in itinere caractérise. Lever ces contraintes constitue autant de défis pour la recherche et l'innovation.

Par exemple, pour les parcelles distantes, il sera nécessaire d'expérimenter les moulins mobiles. Au plan méso-économique, la valorisation des bagasses par l'élevage dépend de la localisation des zones de transformation. Mais si l'industrie cannière et les élevages sont dans les mêmes zones, ils peuvent aussi entrer en compétition pour l'utilisation des tiges sucrées. Ainsi, dans l'hypothèse où les variétés de sorgho sucré se substitueraient aux variétés de sorgho grain, la récolte des tiges pour un usage sucrier pourrait diminuer les superficies mobilisables en vaine pâture. En privilégiant la filière clairin, les producteurs risquent de devoir se séparer d'une partie de leurs animaux. La bagasse de sorgho pourrait certes être valorisée par l'ensilage, mais cela demanderait l'apprentissage de nouveaux procédés, ou leur prise en charge par les industriels ou par un nouvel acteur. Par ailleurs, l'exportation des tiges de sorgho sucré, et la disparition de la vaine pâture qui en résulterait, supprimeraient une restitution de matière organique aux sols. Il faudrait alors compenser ces pertes de fertilité.

$\mathrm{Au}$ plan macro-économique, la promotion du sorgho sucré pourrait avoir des effets bénéfiques à trois niveaux : sur les importations de céréales (pour l'homme et les animaux), sur les importations de viande (la viande est le deuxième poste d'importation) et sur la compétitivité de la filière clairin permettant de réduire les importations d'alcool alimentaire à bas coût. Ces externalités sont des vecteurs potentiels d'accroissement de l'emploi dans le secteur agroindustriel.

L'évaluation in itinere du processus d'innovation lié à l'introduction du sorgho sucré en Haïti confirme l'utilité de faire interagir différentes bases de connaissances et d'expérimentations disciplinaires (technologies et sciences humaines) pour identifier les points de blocage et les opportunités. Elle met en exergue les différentes dimensions techniques, organisationnelles et institutionnelles qui pilotent l'innovation en agriculture. Elle interpelle le besoin d'une politique douanière pour renchérir le prix des produits qui concurrencent la valorisation de la multifonctionnalité du sorgho: maïs importé (pour l'alimentation animale) ou alcools substituts au clairin. L'évaluation réalisée souligne que les variétés de sorgho sucré introduites en 2014 pour tester les potentialités de la multifonctionnalité de cette plante avaient déjà été introduites et adoptées en 1980, sans que les travaux de recherche de l'époque ne permettent d'en tracer l'existence. L'innovation ne résulte donc pas forcément de l'introduction d'une nouveauté dans un système. Elle peut reposer sur la complémentarité des ressources existantes au sein d'un système.

Remerciements. Les auteurs remercient les évaluateurs qui ont apporté des compléments d'information repris dans le texte.

\section{Références}

Benoit-Cattin M. 2016. L'agriculture dans l'économie globale haïtienne : une vue d'ensemble. Chapitre 1. In: Van Vliet G, Pressoir G, Marzin J, Giordano T, eds. Une étude exhaustive et stratégique du secteur agricole/rural haïtien et des investissements publics requis pour son développement. Montpellier: Cirad, $33 \mathrm{p}$. Disponible sur https://agritrop.cirad.fr/580373/7/ID580373.pdf.

Braconnier S, Amaducci S, Basavaraj G, Borges Damasceno C, Clément-Vidal A, Fracasso A, et al., 2014. Sweet sorghum as an alternative energy crop: results from SWEETFUEL project. 22nd European Biomass Conference \& Exhibition, Hamburg, 23-26 June 2014. ISBN 978-88-89407-523.

Chantereau J, Cruz JF, Ratnadass A, Trouche G. 2013. Le sorgho. Versailles: Éditions Quae, CTA, Presses agronomiques de Gembloux, $245 \mathrm{p}$.

Damasceno R, Schaffert E, Dweikat I. 2014. Mining genetic diversity of sorghum as a bioenergy feedstock. In: McCann MC et al., eds. Plants and Bioenergy. Advances in Plant Biology 4: 81-102. DOI: 10.1007/978-1-4614-9329-7 6.

Dehaynin N. 2007. Utilisation du sorgho en alimentation animale. Thèse Université Claude-Bernard, Lyon I, 109 p. Disponible sur www2.vetagro-sup.fr/bib/fondoc/th_sout/dl.php?file= 2007lyon027.pdf.

Dufumier M. 1988. Pénurie alimentaire, agriculture paysanne et politique agricole en Haïti. Économie Rurale 188(1): 26-31.

FAOSTAT, Food and Agriculture Organization of the United Nations. 2016. Available from http://www.fao.org/statistics/fr/.

Freguin S, Devienne S. 2006. Libéralisation économique et marginalisation de la paysannerie en Haïti : le cas de l'Arcahaie. Tiers Monde 187: 621-642.

Leclerc E, Pressoir G, Braconnier S. 2014. L'avenir prometteur du sorgho sucré. Field Actions Science Reports, Special Issue 9. Available from http://factsreports.revues.org/2801.

Maredia MK, Raitzer DA. 2010. Estimating overall returns to international agricultural research in Africa through benefit-cost analysis: a "best-evidence" approach. Agricultural Economics 41 (1): 81-100.

MARNDR. 2012. Synthèse nationale des résultats du Recensement Général de l'Agriculture (RGA) 2008/2009. Port-au-Prince (Haïti): Unité d'Étude et de Programmation, Composante de Statistiques Agricoles.

Paul B. 2012. Institutional change in Haiti, the real challenges. Recherche, Étude et Développement 5: 27-33.

Pierre F. 2005. Identification de créneaux potentiels dans les filières rurales haïtiennes. Les filières canne à sucre. Ministère de l'Agriculture des Ressources Naturelles et du Développement Rural. HA-T1008/ATN-FC-9052.

Pillot D. 1992. Paysans, systèmes et crise: travaux sur l'agraire haïtien. Tome 1: Histoire agraire et développement. Paris: SACAD, 1000 p. ISBN 2-9087104-067, Cirad Montpellier. 
Temple L, Lançon F, Palpacuer F, Paché G. 2011. Actualisation du concept de filière dans l'agriculture et l'agroalimentaire. Économies et Sociétés. Série Systèmes Agroalimentaires 33: 1785-1797.

Temple L, Biénabe E, Barret D, Saint-Martin G. 2016. Methods for assessing the impact of research on innovation and development in the agriculture and food sectors, African Journal of Science, Technology, Innovation and Development 8(5-6): 399-410. DOI: 10.1080/20421338.2016.1219484.

Touzard JM, Temple L, Faure G, Triomphe B. 2014. Systèmes d'innovation et communautés de connaissances dans le secteur agricole et agroalimentaire. Innovations 43: 13-38. DOI: 10.3917/ inno.043.0013.
Trouche G, vom Brocke K, Aguirre S, Chow Z. 2009. Giving new sorghum variety options to resource-poor farmers in Nicaragua through participatory varietal selection. Experimental Agriculture 45(4): 451-467. DOI: 10.1017/S001447970999041X.

Vasilakoglou I, Dhima K, Karagiannidis N, Gatsis T. 2011. Sweet sorghum productivity for biofuels under increased soil salinity and reduced irrigation. Field Crops Research 120: 38-4. DOI: 10.1016/ j.fcr.2010.08.011.

Vinutha KS, Laavanya Rayaprolu K, Yadagiri AV, Umakanth JV, Patil P, Srinivasa R. 2014. Sweet sorghum research and development in India: status and prospects. Sugar Tech 16(2): 133-143. DOI: 10.1007/s12355-014-0302-9.

Citation de l'article : Temple L, Levesque A, Lamour A, Charles D, Braconnier S. 2017. Complémentarité des filières sorgho sucré et canne à sucre en Haïti : évaluation des conditions de développement sectoriel d'une innovation. Cah. Agric. 26: 55006. 